\title{
A murine model of myocardial microvascular thrombosis
}

\author{
Patricia D. Christie, ${ }^{1}$ Jay M. Edelberg, ${ }^{1,2}$ Michael H. Picard, ${ }^{3}$ Andrea S. Foulkes, ${ }^{4}$ \\ Wilfred Mamuya, ${ }^{1,5}$ Hartmut Weiler-Guettler, ${ }^{6}$ Robert H. Rubin, ${ }^{7}$ Peter Gilbert, ${ }^{4}$ \\ and Robert D. Rosenberg ${ }^{1}$
}

\begin{abstract}
${ }^{1}$ Department of Biology, Massachusetts Institute of Technology, Cambridge, Massachusetts 02139, USA
${ }^{2}$ Department of Medicine, Beth Israel Deaconess Medical Center, Boston, Massachusetts 02115, USA

${ }^{3}$ Cardiology Division, Massachusetts General Hospital, Boston, Massachusetts 02124, USA

${ }^{4}$ Department of Biostatistics, Harvard School of Public Health, Boston, Massachusetts 02115, USA

${ }^{5}$ Cardiovascular Division, Brigham and Women's Hospital, Boston, Massachusetts 02115, USA

${ }^{6}$ Blood Research Institute, The Blood Center of Southeastern Wisconsin, Milwaukee, Wisconsin 53226, USA

${ }^{7}$ Center for Experimental Pharmacology and Therapeutics, Harvard-Massachusetts Institute of Technology,

Division of Health Sciences and Technology, Cambridge, Massachusetts 02139, USA

Address correspondence to: Robert D. Rosenberg, Department of Biology 68-480,

Massachusetts Institute of Technology, 77 Massachusetts Avenue, Cambridge, Massachusetts 02139, USA.

Phone: (617) 253-5234; Fax: (617) 258-6553; E-mail: rdrrosen@mit.edu.
\end{abstract}

Received for publication April 20, 1999, and accepted in revised form July 13, 1999.

Disorders of hemostasis lead to vascular pathology. Endothelium-derived gene products play a critical role in the formation and degradation of fibrin. We sought to characterize the importance of these locally produced factors in the formation of fibrin in the cardiac macrovasculature and microvasculature. This study used mice with modifications of the thrombomodulin (TM) gene, the tissue-type plasminogen activator (tPA) gene, and the urokinase-type plasminogen activator (uPA) gene. The results revealed that tPA played the most important role in local regulation of fibrin deposition in the heart, with lesser contributions by TM and uPA (least significant). Moreover, a synergistic relationship in fibrin formation existed in mice with concomitant modifications of tPA and TM, resulting in myocardial necrosis and depressed cardiac function. The data were fit to a statis tical model that may offer a foundation for examination of hemostasis-regulating gene interactions.

J. Clin. Invest. 104:533-539 (1999).

\section{Introduction}

Disorders of hemostasis lead to vascular pathology. Absence of coagulation factors may lead to an increased propensity for bleeding in various vascular beds. Conversely, altered regulation of hemostasis can be associated with a pronounced tendency toward thrombosis in different vascular beds, which can lead to tissue ischemia and infarction. The regulation of this balance is maintained by systematically circulating plasma proteins, membrane-bound proteins, and the endothelium. The propensity of different vascular beds to develop thrombosis may be due to specific disorders of the endothelium. Local control of hemostasis is under the direction of the endothelium, which itself has some diversity in its expression of various gene products $(1,2)$. In mice, differential expression of plasminogen activator inhibitor- 1 has been observed in different tissues, with high levels in aorta, heart, and adipose tissue, and low levels in the liver, adrenals, and kidneys (3). In the mouse, there are higher levels of expression of thrombomodulin (TM) in the abdominal aorta than in the thoracic aorta (4). Endothelial cells derived from microvascular beds express 100 -fold more tissue-type plasminogen activator (tPA) than endothelial cells derived from macrovascular cells (5).

In the Western world, coronary artery thrombosis is usually the result of atherosclerosis. Atherosclerotic plaque rupture is thought to trigger arterial thrombosis mediated by platelets and fibrin, which results in regional ischemia or infarction $(6,7)$. Atherosclerosis is a disease of the macrovasculature (8). Small vessel thrombosis as a result of endothelial cell dysfunction may also produce regional ischemia (9-11). Multiple genes regulate coagulation $(12,13)$. We hypothesized that differential gene expression is important for organ-bed specificity with respect to hemostatic balance. Such a biological system would likely be controlled at the endothelium level (14). We demonstrated previously that the cardiac myocardium regulates the specific activity of its endothelial cells, suggesting a potential role in regulating hemostasis (2). Moreover, fibrin deposition in the cardiac vasculature has a profound effect on heart function; thus, we chose to study this organ bed as a model of thrombus regulation. The use of genetically modified animals provides a powerful tool for unraveling the complex interactions of the various proteins involved in coagulation homeostasis in the cardiac circulation. In this study, we investigated the interaction of 3 endothelium-derived genes TM, tPA, and urokinase-type plasminogen activator (uPA) - and their effects on myocardial thrombosis. TM, a natural anticoagulant, is a membrane-bound protein that facilitates the activation of protein $\mathrm{C}$ to activated protein C (APC) when thrombin is also 
bound to it (15). A primary component of the fibrinolytic system is an inactive zymogen called plasminogen, which, after activation by either tPA or uPA, is able to remove fibrin clots (16). Using genetically modified animals, we studied the relative contributions of the above gene products to the maintenance of the hemostatic balance. We showed that the simultaneous reduction of the natural TM-anticoagulant pathway activity and diminution of tPA-mediated fibrinolytic capacity combined to produce severe tissue damage and functional abnormalities in the hearts of mutant mice. A statistical approach was developed that quantitatively describes the effects of the above hemostatic gene defects, and should aid in detecting genetic interactions that are relevant to the physiology and pathophysiology of the cardiovascular system.

\section{Methods}

Generation of mice. The mice with single-gene modifications $\left(\mathrm{TM}^{+/-}, \mathrm{TM}^{\mathrm{Pro} / \mathrm{Pro}}, \mathrm{TM}^{-/ \text {Pro }}, t \mathrm{PA}^{-/-}\right.$, and $\left.u P A^{-/-}\right)$have all been described previously $(4,17,18)$. Mice deficient in UPA or tPA were obtained from P. Mulligan (Children's Hospital, Boston, Massachusetts, USA). Unless otherwise stated, the background of all mice was mixed C57BL/6 and 129. The wild-type $\left(T M^{+/+}\right)$and $\mathrm{TM}^{+/-}$mice were littermates, and the $\mathrm{F}_{7}$ generation was backcrossed to C57BL/6. The $u P A^{-/-}$and $t P A^{-/-}$mice were backcrossed 4 times to $\mathrm{C} 57 \mathrm{BL} / 6$. The $T M^{-/ P r o}$ lines were generated from a $T M^{\text {Pro/Pro }} \mathrm{F}_{2}$ mouse, and a $\mathrm{TM}^{+/-}$mouse was backcrossed 4 times to $\mathrm{C} 57 \mathrm{BL} / 6\left(\mathrm{~F}_{4}\right)$. The other lines were generated from the $u \mathrm{PA}^{-/-}, t \mathrm{PA}^{-/}$, $T M^{+/-}$, and $T M^{-/ P r o}$ lines as described above. The $t P A^{-/-} T M^{P r o / P r o}$ and $t P A^{-/-} T M^{-/ P r o}$ animals were littermates, as were the $u P A^{-/-} T M^{P r o / P r o}$ and $u P A^{-/-} T M^{-/ P r o}$ animals. The maximum contribution of 129 to the genetic background of the different mouse genotypes ranged from $1.5 \%$ to $12.5 \%$. The genotypes of the animals were identified by lac $Z$ reporter gene analysis of tails and by Southern blot analysis. All these studies were performed on young adult mice (3-6 months of age). We have not noticed any shortening of life-span in animals up to 8 months of age.

Detection of tissue fibrin. Fibrin was measured in mouse tissues by a method that has been described previously (17), with several modifications. The primary antibody was either the monoclonal 59D8 (kindly provided by Marshall Runge, University of Texas Medical Branch at Galveston, Galveston, Texas, USA; ref. 19) or the commercially available mAb NYB T2G1 (Accurate Chemical \& Scientific Corp., Westbury, New York, USA) (20). Protein was detected using a chemiluminescence system (either ECL from Amersham Life Sciences Inc., Arlington, Illinois, USA, or CDP-Star from NEN Life Science Products Inc., Boston, Massachusetts, USA) and was quantitated using a Kodak Image Station (model 440CF; Eastman Kodak Co. Scientific Imaging Systems, New Haven, Connecticut, USA) supplied with 1D Image Analysis Software. Each sample group contained 10 mice, except the following genotypes: $u P A^{-/}-T M^{\text {Pro/Pro }}$ (3 mice), $u P A^{-/-} T M^{-/ P r o}$ (6 mice), $t P A^{-/-} T M^{\text {Pro/Pro }}$ (7 mice), and $u P A^{-/-} t P A^{-/-}$(8 mice).

Statistical analysis. The statistical relationship between fibrin levels and defects in the TM gene and fibrinolytic genes was analyzed using standard linear modeling techniques (21). Plots of fibrin levels within genotypic groups revealed a slight skewness in the data. Therefore, fibrin levels were natural log-transformed $\left(\ln _{e}\right)$ to normalize their distribution; all analyses were performed on these transformed data. A 3-way cellmeans ANOVA model with all pairwise interactions between the TM gene, uPA gene, and tPA gene was first fit to the data, and then $F$ tests were performed to determine whether these factors contributed significantly to the overall fit of the model. In this process, TM was treated as a 4-level factor taking on the values $\mathrm{TM}^{+/+}, \mathrm{TM}^{+/-}, \mathrm{TM}^{\text {Pro/Pro }}$, and $T M^{-/ \text {Pro }}$; uPA and tPA were treated individually as 2-level factors, indicating the presence of the wild-type gene or deletion mutation.

A simple linear regression model was fit to the data. If the potential interactions between the TM gene and the uPA gene, or between the TM gene and the tPA gene, were significant based on the ANOVA results, then all components of these interactions were considered separately in the regression. All main effects were also included. A backward elimination procedure based on Wald tests was performed (22). The final model consisted of all factors with coefficients significantly different from $0(P<0.05)$. Estimated coefficients from the final regression model were reported to indicate the direction and magnitude of the effect of each term on $\ln _{\mathrm{e}}$ fibrin. Unadjusted prediction intervals for multiple comparisons of fibrin levels were also reported for all observed genotype combinations.

Histology. Murine heart sections from 5 wild-type control mice and $3 t P A^{-/-} T M^{-/ P r o}$ animals were prepared for histological analysis as described previously $(17,23)$. Masson's trichrome stain (Sigma HT15; Sigma Diagnostics, St. Louis, Missouri, USA) was performed on alternate sections to those that were stained for fibrin (24).

Cell culture. These studies used both wild-type C57BL/6 and FVB mice. Cardiac microvascular endothelial cells and myocytes were isolated and cultured from murine hearts as described previously (25, 26). The endothelial cells were cultured alone or in the presence of cardiac myocytes, in $12-\mathrm{mm}(0.4-\mu \mathrm{m}$ pore $)$ Transwell dishes (Corning-Costar Corp., Cambridge, Massachusetts, USA), as described previously (2). The cells were cultured for 72 hours, with a medium change at 48 hours. The cultures were then washed twice with PBS, and the medium was changed to DMEM with $1 \%$ BSA and $20 \mathrm{mM}$ glucose, and incubated for an additional 12 hours. The medium was then collected for secreted-protein analysis.

Protein assay. Cellular and secreted protein samples were isolated from cardiac microvascular endothelial cells cultured as described above. Relative antigenic levels of TM were determined by Western dot blotting with 
Table 1

Fibrin deposition in heart

\begin{tabular}{|c|c|c|c|}
\hline Genotype & $\begin{array}{c}\text { Fibrin } \\
\text { deposition } \\
\mathrm{ng} \text { fibrin/ } \\
4 \mathrm{mg} \text { tissue }\end{array}$ & $\begin{array}{c}\text { Fibrin } \\
\text { coefficient } \\
\ln (\mathrm{ng} \text { fibrin/ } \\
4 \mathrm{mg} \text { tissue })\end{array}$ & $\begin{array}{c}\text { Predicted } \\
\text { fibrin level } \\
\ln (\mathrm{ng} \text { fibrin/ } \\
4 \mathrm{mg} \text { tissue }) \\
\text { (95\% prediction interva }\end{array}$ \\
\hline Wild-type & $5.66 \pm 0.57$ & 1.56 & $0.31-2.81$ \\
\hline $\mathrm{TM}^{+/-}$ & $13.5 \pm 2.2$ & 1.08 & $1.39-3.90$ \\
\hline$T M^{\text {Pro/Pro }}$ & $24.1 \pm 5.4$ & 1.28 & $1.58-4.10$ \\
\hline$T M^{-/ P r o}$ & $51.1 \pm 4.9$ & 2.82 & $3.10-5.65$ \\
\hline$t P A^{-/-}$ & $90.4 \pm 11.7$ & 2.95 & $3.25-5.76$ \\
\hline uPA ${ }^{-/-}$ & $45.3 \pm 5.1$ & 2.23 & $2.53-5.04$ \\
\hline \multicolumn{4}{|c|}{ Genotypic crosses } \\
\hline$t P A^{-/-} T M^{+/-}$ & $331 \pm 37$ & NS & $4.34-6.84$ \\
\hline$t P A^{-/-} T M^{\text {Pro/Pro }}$ & $324 \pm 58$ & NS & $4.52-7.05$ \\
\hline$t P A^{-/-} T M^{-/ P r o}$ & $716 \pm 114$ & -0.90 & $5.15-7.70$ \\
\hline$u P A^{-/-} T M^{+/-}$ & $148 \pm 22$ & NS & $3.61-6.12$ \\
\hline uPA ${ }^{-/-} T M^{\text {Pro/Pro }}$ & $42.9 \pm 5.5$ & -1.32 & $2.34-5.15$ \\
\hline$u P A^{-/-} \mathrm{TM}^{-/ P r o}$ & $163 \pm 73$ & -1.87 & $3.42-6.05$ \\
\hline$t P A^{-/-} u P A^{-/-}$ & $353 \pm 51$ & -1.02 & $4.44-6.99$ \\
\hline
\end{tabular}

Column 2 is the average calculated for the fibrin deposition in each genotypic group. The values are average \pm SD. Column 3 contains the coefficients from the linear regression analysis of the fibrin deposition values for all of the terms. The unadjusted $95 \%$ prediction intervals for fibrin levels are reported in column 4. NS, not significant.

rat anti-mouse TM (a gift from S. Kennel, University of Tennessee, Oak Ridge, Tennessee, USA) at a dilution of 1:1,000. Rabbit anti-mouse uPA and rabbit anti-mouse tPA were obtained from American Diagnostica Inc. (Greenwich, Connecticut, USA) and were used at 1:1,000 dilutions. Peroxidase-labeled donkey polyclonal antibodies to rat and rabbit IgG (Jackson ImmunoResearch Laboratories Inc., West Grove, Pennsylvania, USA) were used as secondary antibodies, at a dilution of 1:1,000. Blots were developed with a chemiluminescence system (ECL; Amersham Life Sciences Inc.). All protein analyses were performed in triplicate sets, with a minimum of 2 independent experiments.

Echocardiography. Selected mice genotypes (3-6 animals of each type) were chosen for echocardiography (see Table 3). The mice were anesthetized with $0.3 \mathrm{~mL}$ of $2.5 \%$ Avertin and secured to an imaging table that allowed variable degrees of the decubitus position. The chest hair was removed, and electrocardiogram leads were placed on 3 limbs of each mouse. M-mode and 2dimensional transthoracic echocardiography (using a SONOS 5500 machine from Hewlett-Packard, Andover, Massachusetts, USA) was performed with a broad-band, phased-array ultrasound transducer with a frequency range of 5-12 MHz. The instrument settings were optimized to allow maximum frame rates $(120 \mathrm{~Hz})$ and best left ventricular (LV) endocardial visualization. Two-dimensional and M-mode images were obtained at midpapillary level with a short-axis view. High-frame-rate images were digitally captured and stored on magneto-optical disk for off-line analysis. The images were reviewed to assess regional LV function. Heart rates were measured from cycle lengths on the M-mode echocardiograms. Electronic calipers were used to measure the 2-dimensional images for $\mathrm{LV}$ internal diameter (LVID) at end-diastole and end-systole; anterior and posterior wall thickness at end-diastole; and short-axis endocardial area at end-diastole and end-systole (27). At least 5 measurements were made for each parameter and averaged. Fractional shortening (derived from the LVID measurements) and fractional area change (derived from the area measurements) were used as measures of LV systolic function.

Regional wall motion was quantitated on the midventricular short-axis image by modification of the standard wall motion scoring system. Using the insertion points of the right ventricular free wall to define the interventricular septum, this image was divided into 4 equal myocardial segments corresponding to the anterior, septal, posterior, and lateral regions. Myocardial segments were assigned the following scores: hyperkinetic segments, 0; normal moving segments, 1; hypokinetic segments, 2; and akinetic segments, 3. The wall motion score was expressed as a total score ranging from 0 to 12 .

\section{Results}

Fibrin deposition in mutant mice. The fibrin Western blot assay quantitated the amount of fibrin present in each heart (Table 1, column 2). When the number of fully functional TM alleles was gradually reduced from the wild-type (in $T M^{+/-}, T M^{\text {Pro/Pro }}$, and $T M^{-/ P r o}$; ref. 17), the concentration of fibrin increased (from 5.66 in the wild-type to $13.5,24.1$, and $51.1 \mathrm{ng}$ fibrin/4 $\mathrm{mg}$ tissue, respectively). Among the single-knockout animals, $t P A^{-/-}$had the highest concentration of fibrin (90.4 $\mathrm{ng}$ fibrin/4 mg tissue). The levels of fibrin in doubleknockout animal models also increased, with the highest deposition observed when $t P A^{-/-}$was combined with the TM-modified animals. The $t P A^{-/-} \mathrm{TM}^{-/ \text {Pro }}$ mice had the most fibrin (716 $\mathrm{ng}$ fibrin/4 $\mathrm{mg}$ tissue) of all

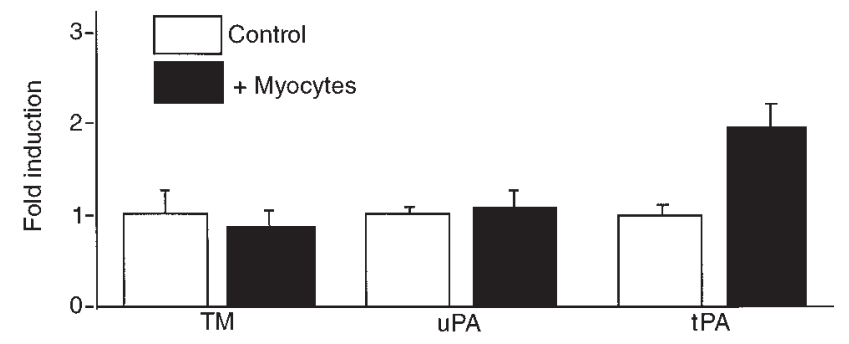

Figure 1

Cardiac microvascular endothelial cell and cardiac myocyte culture. Protein analysis of the levels of TM, UPA, and TPA from cardiac microvascular endothelial cells cultured alone (open bars) and in the presence of cardiac myocytes (filled bars). 

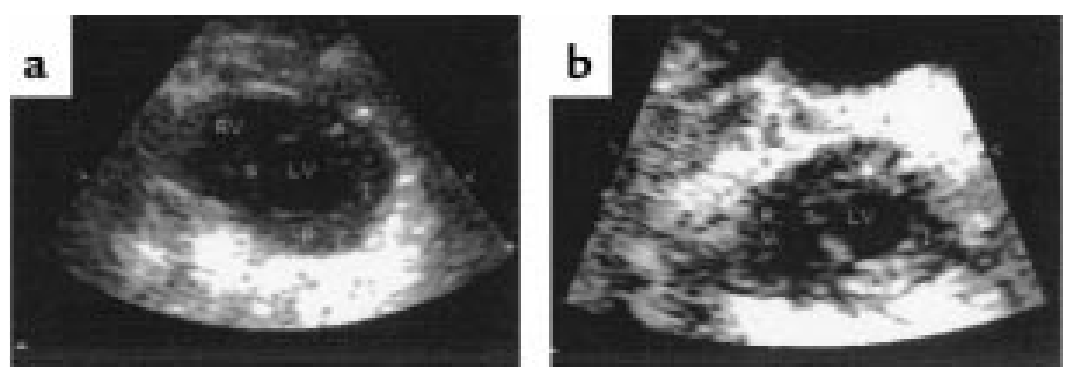

Figure 2

Echocardiography: short-axis view of mouse heart at end-diastole. (a) TM-/Pro animal. (b) $t P A^{-/}-T M^{-/ P r o}$ animal. RV, right ventricular cavity; LV, left ventricular cavity; a, anterior myocardial segment; $s$, septal segment; $p$, posterior segment; I, lateral segment.

the animals examined. The combination of $u \mathrm{PA}^{-/-}$and the TM-modified animals produced a modest increase in fibrin deposition (ranging from 42.9 to $163 \mathrm{ng}$ fib$\mathrm{rin} / 4 \mathrm{mg}$ tissue).

The 3-way ANOVA table with all pairwise interactions is given in Table 2. The test statistic for the overall $F$ test was $50.64(P=0.0001)$, indicating that not all coefficients in the model equaled $0 . F$ tests for the interactions between TM and uPA $(F=10.41, P=0.0001)$, $\mathrm{TM}$ and $\mathrm{tPA}(F=3.44, P=0.020)$, and $\mathrm{tPA}$ and $\mathrm{uPA}(F=$ $4.20, P=0.043)$ revealed that all pairwise interactions contributed in a statistically significant manner to the variability in $\ln _{\mathrm{e}}$ fibrin deposition.

The results of fitting the final regression model to the data are shown in Table 1 , column 3. Interactions between $\mathrm{TM}^{+/-}$and $u \mathrm{PA}^{-/-}, \mathrm{TM}^{+/-}$and $t \mathrm{PA}^{-/-}$, and $\mathrm{TM}^{\text {Pro/Pro }}$ and $t P A^{-/-}$were not significant, and were removed from the final model. The parameter estimates given in Table 1 are estimates of the coefficients for the variables (referred to as fibrin coefficients) in the final model. Predictions for new observations can be made using the equation below, where Pred $[\ln ($ Fibrin $)]$ is the predicted $\ln _{\mathrm{e}}$ fibrin value for a new observation:

Pred $[\ln ($ Fibrin $)]=1.56+1.08\left(T^{+/-}\right)+1.28\left(T^{\text {Pro/Pro }}\right)+$ $2.82\left(\mathrm{TM}^{-/ P r o}\right)+2.23\left(u P A^{-/-}\right)+2.95\left(\right.$ PPA $\left.^{-/}\right)-$

1.32( $\left.U P A^{-/-} T^{\text {Pro/Pro }}\right)-1.87\left(U P A^{-/}-T^{-/ P r o}\right)-$ $0.90\left(t P A^{-/-} T^{-/ P r o}\right)-1.02\left(u P A^{-/-t P A^{-/-}}\right)$.

Based on this model, it appears that the relationships of the fibrin coefficients for prediction of fibrin levels between $u P A^{-/-}$, some of the TM genotypes, and $t P A^{-/-}$ are not simply additive. In general, the effect of having 2 modifications/knockouts is less than the sum of the effects of having either one. In other words, the predicted fibrin level in a mouse that is both $u \mathrm{PA}^{-/-}$and $T M^{\text {Pro/Pro }}$, for example, is not simply the sum of the predicted levels of a $u \mathrm{PA}^{-/-}$ mouse and a $T M^{\text {Pro/Pro }}$ mouse. The levels of fibrin are most greatly affected when TM is modified and tPA is removed in a single animal.

Unadjusted 95\% prediction intervals for each observed genotype are given in Table 1, column 4 (we are 95\% confident that the $\ln _{\mathrm{e}}$ fibrin level for a new observation with the corresponding genotype will fall within these ranges).
Cardiac myocyte induction of tPA. Cardiac microvascular endothelial cells were cultured alone and in the presence of cardiac myocytes. Cardiac myocytes induced increases in endothelial levels of tPA to $191 \pm 13 \%$ of baseline values (Figure 1). The communication between the cardiac myocytes and the endothelial cells did not alter TM or uPA levels ( $92 \pm 15 \%$ and $103 \pm 12 \%$, respectively, compared with baseline values). Levels of TM, tPA, and uPA in cardiac myocytes in monoculture were not detectable.

Echocardiography. A subset of experimental mice was subjected to echocardiography (Table 3). This subset consisted of wild-type $\left(\mathrm{TM}^{+/+}\right), \mathrm{TM}^{+/-}, \mathrm{TM}^{-/ P r o}, t \mathrm{PA}^{-/-}$, and $t P A^{-/-} T M^{-/ P r o}$ animals. Two representative echocardiograms of the mice are shown in Figure 2. No effect on cardiac function was seen when TM was mutated to drastically reduce its anticoagulant function $\left(\mathrm{TM}^{+/-}\right.$ and $\left.T M^{-/ P r o}\right)$. A global decrease in cardiac function was observed in $t P A^{-/-}$mice. When $t P A^{-/-} T M^{-/ P r o}$ mice were examined, they were found to have regional myocardial dysfunction suggestive of myocardial infarcts. Examination of the echocardiograms demonstrates that the infarction was localized to the LV free wall in these animals.

Heart histology of $t \mathrm{PA}^{-/-} \mathrm{TM}^{-/ P r o}$ mice. The fibrin Western blot provides a quantitative description of how much fibrin is present in the heart tissue; immunohistochemistry shows where the fibrin is (Figure 3). Comparison of hearts of $t \mathrm{PA}^{-/-} \mathrm{TM}^{-/ P r o}$ mice and wild-type controls showed that fibrin is present in both macrovasculature (Figure 3a) and microvasculature (not shown).
Table 2

ANOVA table of the genotype interactions

\begin{tabular}{lccccc}
\hline Source & DF & Sum of squares & Mean square & $F$ value & $\operatorname{Pr}>F$ \\
Model & 12 & 230.408 & 19.201 & 50.64 & 0.0001 \\
Error & 103 & 39.050 & 0.379 & & \\
Corrected total & 115 & 269.458 & & &
\end{tabular}

\begin{tabular}{lccccc}
\multicolumn{1}{c}{ Source } & DF & Sum of squares & Mean square & $F$ value & $\operatorname{Pr}>F$ \\
TM & 3 & 23.87 & 7.957 & 20.99 & 0.0001 \\
uPA & 1 & 8.942 & 8.942 & 23.59 & 0.0001 \\
tPA & 1 & 52.246 & 52.246 & 137.8 & 0.0001 \\
TM $\times$ tPA & 3 & 3.91 & 1.303 & 3.44 & 0.0196 \\
TM $\times$ uPA & 3 & 11.845 & 3.948 & 10.41 & 0.0001 \\
uPA $\times$ tPA & 1 & 1.591 & 1.591 & 4.2 & 0.043 \\
\hline
\end{tabular}

DF, degrees of freedom; Pr, probability. 

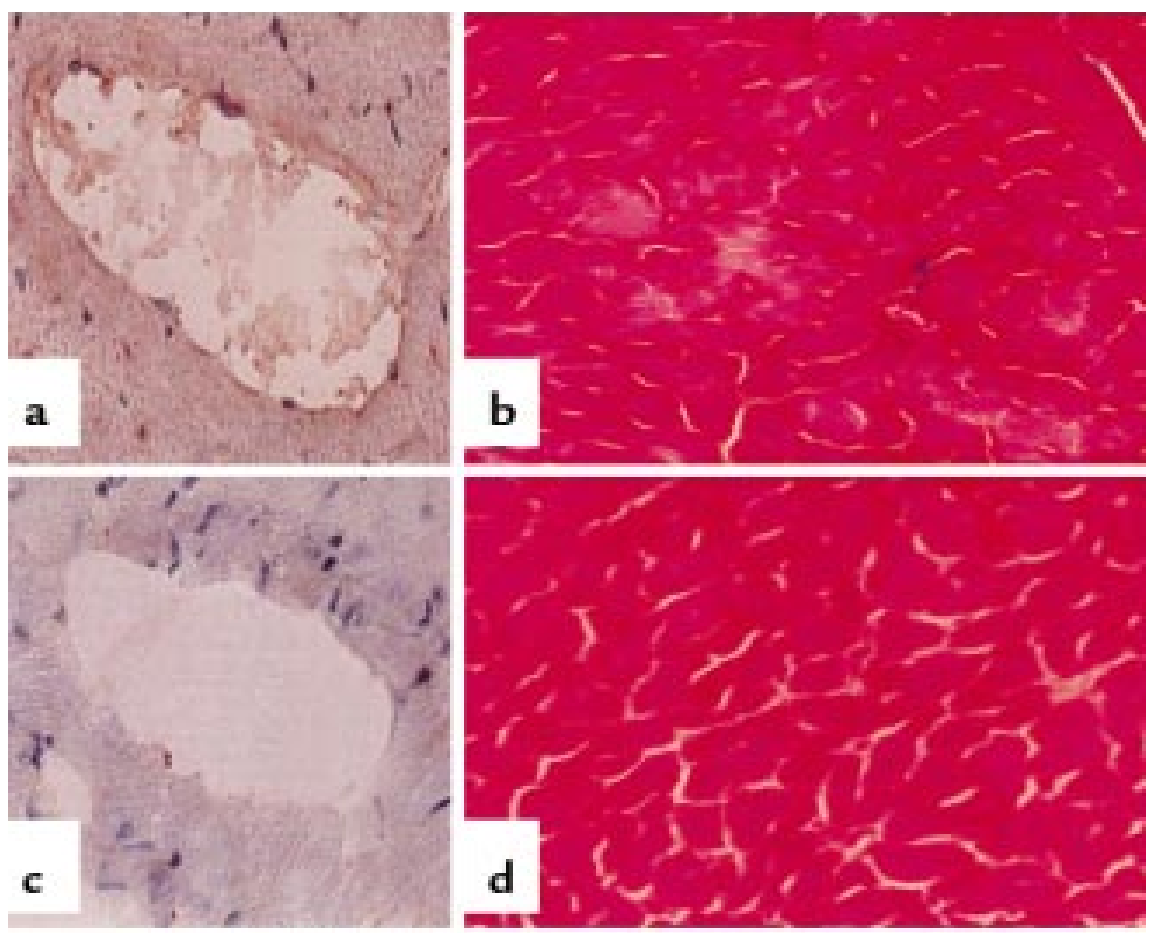

\section{Figure 3}

Histology of heart. (a and $\mathbf{c}$ ) Fibrin immunohistochemistry. Frozen tissue sections of $t P A^{-/-} \mathrm{TM}^{-/ \text {Pro }}(\mathbf{a})$ and wildtype (c) mice were fixed in acidic formalin to wash out soluble fibrinogen/fibrin; reacted with the fibrinogen/fibrin-specific antibody; and counterstained with hematoxylin. The dark-brown horseradish peroxidase reaction product shows fibrin present in the macrovasculature. (b and d) Trichrome stain. Frozen tissue sections of $t P A^{-/-} T M^{-/ P r o}(\mathbf{b})$ and wildtype (d) mice were processed according to standard protocols (24). The $t P A^{-/-} \mathrm{TM}^{-/ P r o}$ section shows myocardial infarct caused by the fibrin present in distal arteries. This tissue death is not present in the wild-type hearts. All images are sections from the LV wall of the heart. $\times 150$.

When the heart was examined distal to the area of intravascular fibrin deposition, abnormalities were observed in the trichrome stain of the $t P A^{-/-} T M^{-/ P r o}$ mice but not the wild-type mice. Trichrome stains use 3 different dyes to identify and distinguish nuclei (blue to black), collagen (blue), and cytoplasm and muscle (red). When muscle necrosis occurs, fibers invade the necrotic tissue; these fibers are stained with aniline blue. The trichrome stain shows clearly that myocardial infarction is present in the $\mathrm{LV}$ myocardium of the $t P A^{-/-} T M^{-/ P r o}$ animals. On average, 1 area of muscle necrosis was present in each randomly chosen section of the heart.

\section{Discussion}

The heart is an organ in which there is heterogeneity in gene expression in the endothelium (2). Recent data have suggested that tPA is associated only with a distinct subpopulation of the endothelial cells (28), and that the level of tPA can be increased when these cells are exposed to pertinent stimuli (29). The tissues with the highest levels of tPA are the brain and heart (E.G. Levin and K.G. Osborn, unpublished observations; also reported in ref. 29). The tissue with the highest expression level of uPA is the kidney (30). TM has high levels of expression in the vascularized tissues, including the heart $(4,31)$.

The fibrin Western blot illustrates the effect of selective gene modification on hemostasis in different vascular beds. Statistical analysis provides a tool for examining the interactions and effects of various gene deletions/modifications in an in vivo mouse model. The combination of modified TM and a gene deletion in the fibrinolytic pathway greatly increases the levels of fibrin. In the animals with a single knockout/modification, removal of uPA causes the same magnitude of effect on fibrin deposition as is seen in $T M^{-/ P r o}$ mice. Both uPA and TM have a more general role in the fibrinolytic/coagulation cascade than tPA; only tPA has specificity for the removal of fibrin from the vascular system (16). The removal of tPA appears to have the greatest effect among the animals with a single knockout/modification. This is also reflected in $t P A^{-/-}$mice having a larger fibrin coefficient than either $T M^{-/ P r o}$ or $u \mathrm{PA}^{-/-}$mice. It has been reported (32) that tPA and uPA are able to compensate for one another. This could contribute to the lower levels of fibrin present in the single knockouts. When the $t P A^{-/}$characteristic is combined with the $T M^{-/ P r o}$ modification, the resulting $t P A^{-/-} T M^{-/ P r o}$ mutant mouse has fibrin levels that can be calculated by adding the fibrin coefficients (predicted $\ln _{\mathrm{e}}$ fibrin levels) of the 2 original mutants to the wild-type coefficient (no additional interaction is observed when the 2 genes are modified or deleted). The predicted $\ln _{\mathrm{e}}$ fibrin level of a $t P A^{-/-} T M^{\text {Pro/Pro }}$ mouse is 5.79, which is simply the sum of the individual $\ln _{e}$ fibrin levels of a wild-type (1.56), a $t P A^{-\digamma^{-}}$(2.95), and a $T M^{\mathrm{Pro} / \mathrm{Pro}}(1.28)$ mouse. When uPA is removed in the presence of the modified TM, it appears that tPA is able to compensate, producing a negative fibrin coefficient for the interaction of UPA and TM. The predicted $\ln _{\mathrm{e}}$ fibrin level for a $u P A^{-/-} T M^{-/ P r o}$ mouse is 4.74 , which is less than the sum of the individual $\ln _{\mathrm{e}}$ fibrin coefficients of a wild-type (1.56), a $T M^{-/ P r o}$ (2.82), and a $u P A^{-/-}$(2.23) mouse, which is 6.61. The fibrin levels of the mice with 2 genetic modifications were higher than either of the single knockouts, but not as high as the $t P A^{-/-} T M^{P r o} /$ Pro mice. Because of the number of mouse lines used in this study, it was not always possible to genetically match the backgrounds of all the 
Table 3

Echocardiographic measurements

\begin{tabular}{|c|c|c|c|c|c|}
\hline Genotype & Sample size & LVID dias (cm) & Anterior wall $(\mathrm{cm})$ & Wall motion score & LV function \\
\hline Wild-type & 6 & $0.237 \pm 0.004$ & $0.091 \pm 0.009$ & $4.0 \pm 0.0$ & Normal \\
\hline $\mathrm{TM}^{+/-}$ & 3 & $0.301 \pm 0.012$ & $0.079 \pm 0.005$ & $4.0 \pm 0.0$ & Normal \\
\hline$T M^{-/ P r o}$ & 3 & $0.302 \pm 0.0333$ & $0.079 \pm 0.007$ & $4.0 \pm 0.0$ & Normal \\
\hline$t P A^{-/-}$ & 3 & $0.333 \pm 0.053$ & $0.070 \pm 0.003$ & $6.7 \pm 0.6$ & Global decrease \\
\hline$t P A^{-/-} T M^{-/ P r o}$ & 5 & $0.289 \pm 0.038$ & $0.084 \pm 0.006$ & $5.2 \pm 0.5$ & Regional decrease \\
\hline
\end{tabular}

All numbers are expressed as averages \pm SD. LVID dias, left ventricular cavity inner diastole diameter. There are no statistical differences seen in any of the measurements done except for the wall motion scores. There are significant statistical differences between each of the wild-type, and $T M^{-/ P r o}$ with $t P A^{-/-}(P \leq 0.001)$; between each of the wild-type, $T M^{+/-}$, and $T M^{-/ P r o}$ with $t P A^{-/-} T M^{-/ P r o}(P \leq 0.001)$; and between $t P A^{-/-}$and $t P A^{-/-} T M^{-/ P r o}(P \leq 0.05)$.

animals by using littermates as wild-type controls. There are strain-specific differences reported for knockouts of fibronectins (33), fibrinogen (34), and other genes (35) that are dependent on the genetic background of the mouse (summarized in ref. 35). In this study, there was a population-based contribution of 129 to the genetic background of the mice, but because the distribution of fibrin deposition was similar for each of the different mouse lines used, the potential contribution of genetic modifiers to a specific mouse line should be minimal. In fact, in over 2 years of sibling/sibling breedings of the $T M^{\text {Pro/Pro }}$ mice, we have not observed any increase in fibrin deposition in the vascular beds cited in this study. Thus, although we cannot rule out that the magnitude of some of the results may have been influenced by slight variations in the background of some of the lines, our experience with the $T M^{\text {Pro/Pro }}$ mice supports the conclusions put forth in this manuscript.

The data from this study offer potential insight into the complex interdependence of the coagulation and fibrinolytic pathway components. We demonstrated previously that the $T M^{\text {Pro }}$ mutation has suppressed activated protein $\mathrm{C}$ (APC) generation. However, the Pro mutant protein maintains the ability to bind thrombin, albeit with decreased affinity, suggesting that non-APC pathways may be affected by the thrombin/TM Pro mutant complex (17). The data in this study demonstrate that in the tPA-knockout mice, the $T M^{\text {Pro }}$ mutation has effects on fibrin deposition similar to the TM-null allele, suggesting that APC generation is critical in this genotype. However, in the uPA-knockout mice, the $T M^{\text {Pro }}$ mutation makes a minimal contribution to overall fibrin levels compared with the TM deletion. These data suggest that in $u \mathrm{PA}^{-/-}$mice, a potential $\mathrm{TM} /$ thrombin complex may affect other non-APC-dependent targets. Previous studies have demonstrated that the TM/thrombin complex regulates the rate of plasmin generation through the effects of thrombin-activated fibrinolysis inhibitor (TAFI) on both tPA and UPA (36-39). In addition, the $\mathrm{TM} /$ thrombin interaction has been shown to directly modulate the uPA receptor (40). While these interactions may not account for our current observations, we propose that additional, as yet undescribed, molecular interactions may contribute to the interactions between TM and plasminogen activator pathways.
The above data indicate that tPA is important for normal heart function. We demonstrated previously that other genes are critical to cardiac development and physiology (including VEGF and Flk-1), and are regulated by communication between cardiac myocytes and the microvascular endothelium (2). The present studies suggest that levels of tPA may be regulated by a similar mechanism. It was shown that in the coculture of endothelial cells and myocytes, levels of tPA increased, whereas the UPA and TM protein levels were not affected. It has been suggested (32) that tPA is important for dissolving embolized clots, whereas uPA is more necessary for the healing of wound fields on the skin. Any disruption in communication between the cardiac myocytes and the endothelium could potentially lead to altered levels in tPA, possibly resulting in profound effects on fibrin levels. As judged by echocardiography, the hearts of the animals with the TM defect function normally, while the tPAdeficient animals have global defects in their cardiac function. Because tPA is expressed throughout the heart, it is not surprising that its removal leads to a global cardiac effect (28). The $t P A^{-/-} T M^{-/ P r o}$ mice exhibited a regional effect, reflected in $\mathrm{LV}$ dysfunction. In addition, these mice displayed indications of focal myocardial necrosis that correlate with dramatically increased amounts of fibrin deposited in the myocardial vasculature. We are currently examining larger numbers of animals to develop a model in which gene interactions are related to cardiac function.

Echocardiography is an excellent technique for looking at cardiac function of genetically modified mice. It has been shown in this study that global and regional cardiac defects can be determined noninvasively. This technique could potentially be used as an evaluation tool in genetic manipulation studies of cardiac abnormalities. Because of the noninvasive nature of the technique, it would be of great benefit in studying gene-modified animals as a disease progresses. For instance, we have previously reported an age-related dependence of fibrin deposition in TMnull chimeric mice (41). Echocardiography would provide a means for observing the physiological consequences of the progression of this disease state.

In conclusion, we have produced an in vivo mouse model with lesions associated with microvascular thrombosis. The $t P A^{-/-} T M^{-/ P r o}$ animals have $\mathrm{LV}$ region- 
al wall motion abnormalities, as demonstrated using echocardiography. This dysfunction is likely the result of the overwhelming extent of fibrinous vascular occlusion in the hearts of these animals. Thus, it appears that the addition of modified TM is sufficient to convert the biochemically defined hyperfibrinolytic state and global cardiac dysfunction observed in tPA-deficient mice into a mutant mouse with focal myocardial tissue necrosis associated with LV dysfunction. The $t P A^{-/-} T M^{-/ P r o}$ mouse should prove to be a good in vivo model of myocardial infarction caused by thrombosis.

\section{Acknowledgments}

We would like to thank M. Lech and A. Israel for maintaining the mouse colony. This work was supported by National Institutes of Health grants PO1-41484 (to R.D. Rosenberg and P.D. Christie), HL-41484 (to R.D. Rosenberg), HL-07604 (to W. Mamuya), and T32AI07358 (to A.S. Foulkes), and by American Heart Association Fellowship Award 13-419-967 (to J.M. Edelberg).

1. Aird, W.C., et al. 1997. Vascular bed-specific expression of an endothelial cell gene is programmed by the tissue microenvironment. J. Cell Biol. 138:1117-1124

2. Edelberg, J.M., et al. 1998. PDGF mediates cardiac microvascular communication. J. Clin. Invest. 102:837-843.

3. Sawdey, M.S., and Loskutoff, D.J. 1991. Regulation of murine type 1 plasminogen activator inhibitor gene expression in vivo. Tissue specificity and induction by lipopolysaccharide, tumor necrosis factor-alpha, and transforming growth factor-beta. J. Clin. Invest. 88:1346-1353.

4. Weiler-Guettler, H., Aird, W.C., Husain, M., Rayburn, H., and Rosenberg, R.D. 1996. Targeting of transgene expression to the vascular endothelium of mice by homologous recombination at the thrombomodulin locus. Circ. Res. 78:180-187.

5. Speiser, W., Anders, E., Preissner, K.T., Wagner, O., and Müller-Berghaus, G. 1987. Differences in coagulant and fibrinolytic activities of cultured human endothelial cells derived from omental tissue microvessels and umbilical veins. Blood. 69:964-967.

6. Dalager-Pedersen, S., Ravn, H.B., and Falk, E. 1998. Atherosclerosis and acute coronary events. Am. J. Cardiol. 82:37T-40T.

7. Fuster, V. 1994. Lewis A. Conner Memorial Lecture. Mechanisms leading to myocardial infarction: insights from studies of vascular biology [erratum 1995, 91:256]. Circulation. 90:2126-2146.

8. Epstein, F. 1999. Atherosclerosis: an inflammatory disease. N. Engl. J. Med. 340:115-135.

9. Verdouw, P.D., van den Doel, M.A., de Zeeuw, S., and Duncker, D.J. 1998. Animal models in the study of myocardial ischaemia and ischaemic syndromes. Cardiovasc. Res. 39:121-135.

10. Jonker, J.J.C., and Chesebro, J.H. 1992. Chronic coronary disease. In Thrombosis in cardiovascular disorders. V. Fuster and M. Verstraete, editors. W.B. Saunders Co. Philadelphia, PA. 363-373.

11. Selwyn, A.P., Kinlay, S., Craeger, M., Libby, P., and Ganz, P. 1997. Cell dysfunction in atherosclerosis and ischemic manifestations of coronary artery disease. Am. J. Cardiol. 97:17-23.

12. Esmon, C.T. 1993. Cell mediated events that control blood coagulation and vascular injury. Annu. Rev. Cell Biol. 9:1-26.

13. Rock, G., and Wells, P. 1997. New concepts in coagulation. Crit. Rev. Clin. Lab. Sci. 34:475-501.

14. Cines, D.B., et al. 1998. Endothelial cells in physiology and in the pathophysiology of vascular disorders. Blood. 91:3527-3561.

15. Esmon, C.T., and Owen, W.G. 1981. Identification of an endothelial cell cofactor for thrombin-catalyzed activation of protein C. Proc. Natl. Acad. Sci. USA. 78:2249-2252.

16. Collen, D., and Lijnen, H.R. 1991. Basic and clinical aspects of fibrinolysis and thrombolysis. Blood. 78:3114-3124.
17. Weiler-Guettler, H., et al. 1998. A targeted point mutation in thrombomodulin generates viable mice with a prethrombotic state. J. Clin. Invest. 101:1983-1991.

18. Carmeliet, P., et al. 1994. Physiological consequences of loss of plasminogen activator gene function in mice. Nature. 368:419-424.

19. Hui, K.Y., Haber, E., and Matsueda, G.R. 1983. Monoclonal antibodies to a synthetic fibrin-like peptide bind to human fibrin but not fibrinogen. Science. 222:1129-1132.

20. Kudryk, B., Rohoza, A., Ahadi, M., Chin, J., and Wiebe, M.E. 1984. Specificity of a monoclonal antibody for the NH2-terminal region of fibrin. Mol. Immunol. 21:89-94.

21. Seber, G.A.F. 1977. Linear regression analysis. John Wiley \& Sons. New York, NY. 125-139.

22. Christensen, R. 1987. Plain answers to complex questions: the theory of linear models. Springer-Verlag. New York, NY. 45-78.

23. Dvorak, H.F., Form, D.M., Manseau, E.J., and Smith, B.D. 1984. Pathogenesis of desmoplasia. I. Immunofluorescence identification and localization of some structural proteins of line 1 and line 10 guinea pig tumors and of healing wounds. J. Natl. Cancer Inst. 73:1195-1205.

24. Prophet, E.B., Mills, B., Arlington, J.B., and Sobrin, L.E. 1994. Laboratory methods in histotechnology. American Registry of Pathology. Washington, DC. 132-133.

25. Lodge, P.A., Haisch, C.E., and Thomas, F.T. 1992. A simple method of vascular endothelial cell isolation. Transplant. Proc. 24:2816-2817.

26. Nishida, M., Springhorn, J.P., Kelly, R.A., and Smith, T.W. 1993. Cell-cell signaling between adult rat ventricular myocytes and cardiac microvascular endothelial cells in heterotypic primary culture. J. Clin. Invest. 91:1934-1941.

27. Scherrer-Crosbie, M., et al. 1999. Three-dimensional echo assessment of left ventricular wall motion abnormalities in mouse myocardial infarction. J. Am. Soc. Echocardiogr. In press.

28. Levin, E.G., Santell, L., and Osborn, K.G. 1997. The expression of endothelial tissue plasminogen activator in vivo: a function defined by vessel size and anatomic location. J. Cell Sci. 110:139-148.

29. Levin, E.G., Osborn, K.G., and Schleuning, W.D. 1998. Vessel-specific gene expression in the lung: tissue plasminogen activator expression is limited to bronchial arteries and pulmonary vessels of discrete size. Chest. 114(Suppl. 1):68S

30. Wagner, S.N., et al. 1996. Sites of urokinase-type plasminogen activator expression and distribution of its receptor in the normal human kidney. Histochem. Cell Biol. 105:53-60.

31. Ford, V.A., Stringer, C., and Kennel, S.J. 1992. Thrombomodulin is preferentially expressed in Balb/c lung microvessels. J. Biol. Chem. 267:5446-5450.

32. Bugge, T.H., et al. 1996. Urokinase-type plasminogen activator is effective in fibrin clearance in the absence of its receptor or tissue-type plasminogen activator. Proc. Natl. Acad. Sci. USA. 93:5899-5904.

33. George, E.L., Baldwin, H.S., and Hynes, R.O. 1997. Fibronectins are essential for heart and blood vessel morphogenesis but are dispensable for initial specification of precursor cells. Blood. 90:3073-3081.

34. Suh, T.T., et al. 1995. Resolution of spontaneous bleeding events but failure of pregnancy in fibrinogen-deficient mice. Genes Dev. 9:2020-2033

35. Erickson, R.P. 1996. Mouse models of human genetic disease: which mouse is more like a man? Bioessays. 18:993-998.

36. Bajzar, L., Nesheim, M., Morser, J., and Tracy, P.B. 1998. Both cellular and soluble forms of thrombomodulin inhibit fibrinolysis by potentiating the activation of thrombin-activable fibrinolysis inhibitor. J. Biol. Chem. 273:2792-2798.

37. Bajzar, L., Nesheim, M.E., and Tracy, P.B. 1996. The profibrinolytic effect of activated protein $\mathrm{C}$ in clots formed from plasma is TAFI-dependent. Blood. 88:2093-2100.

38. Hosaka, Y., Takahashi, Y., and Ishii, H. 1998. Thrombomodulin in human plasma contributes to inhibit fibrinolysis through acceleration of thrombin-dependent activation of plasma procarboxypeptidase B. Thromb. Haemost. 79:371-377.

39. Nesheim, M. 1998. Fibrinolysis and the plasma carboxypeptidase. Curr. Opin. Hematol. 5:309-313.

40. Wilhelm, S., Wilhelm, O., Schmitt, M., and Graeff, H. 1994. Inactivation of receptor-bound pro-urokinase-type plasminogen activator (pro-uPA) by thrombin and thrombin/thrombomodulin complex. Biol. Chem. Hoppe Seyler. 375:603-608.

41. Healy, A.M., Hancock, W.W., Christie, P.D., Rayburn, H.B., and Rosenberg, R.D. 1998. Intravascular coagulation activation in a murine model of thrombomodulin deficiency: effects of lesion size, age, and hypoxia on fibrin deposition. Blood. 92:4188-4197. 